\title{
Frailty in level 1 patients in Northumbria Healthcare NHS Foundation Trust
}

\author{
Authors: Avinash Aujayeb ${ }^{A}$ and Ellen Tullo ${ }^{A}$
}

\section{Introduction}

There is a research gap with regards to supporting the use of the clinical frailty score (CFS) in the acute management of COVID-19 patients. The COPE study assessed the effect of frailty on outcomes in people of all ages with COVID-19 and showed that frailty increases risk of mortality, after accounting for age and comorbidities. ${ }^{1}$

\section{Method}

We performed a retrospective analysis of all patients with COVID-19 patients who did not require respiratory support (continuous positive airway pressure (CPAP) / bilevel positive airway pressure (BiPAP)), so called 'level 1' patients. Basic demographics, comorbidities, outcomes and CFS were collected. All electronic notes reviewed by a care of the elderly consultant to independently verify the CFS. Descriptive statistical methodology was applied.

\section{Results}

Four-hundred and two patients were identified. Median age was 78.5 years (range 19-100; interquartile range 16). The prevalence of frailty, defined as CFS $5-8$ was $48 \%(n=193)$. Overall mortality was $27 \%$, and mortality rate in that group was $42 \%(n=81)$. CFS had a linear relationship with CFS (Fig 1).

\section{Conclusions}

Our mortality rates are in line with other studies and our data supports the use of CFS in the decision-making process and assessment of COVID-19 patients. Our data are limited by noninclusion of the level 2/3 patients and correction of other variables.

\section{Conflicts of interest}

None declared.

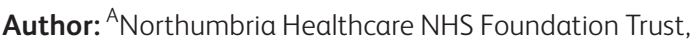
Northumbria, UK

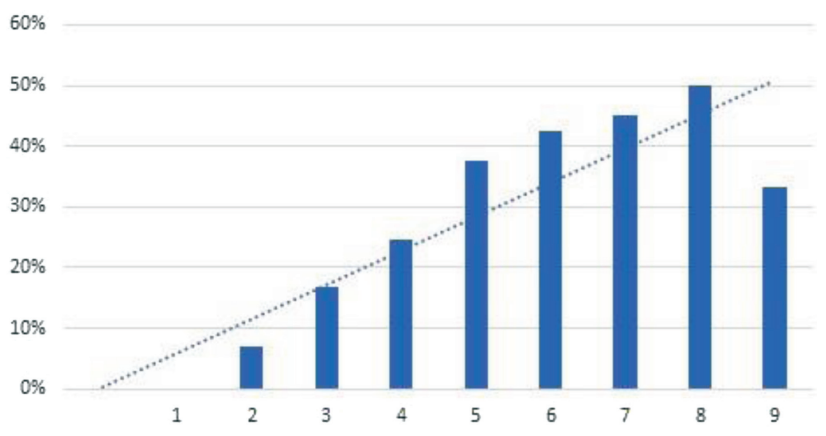

Fig 1. Northumbria Healthcare NHS Foundation Trust mortality rate per clinical frailty score.

\section{Reference}

1 Hewitt J, Carter B, Vilches-Moraga A et al. The effect of frailty on survival in patients with COVID-19 (COPE): a multicentre, European, observational cohort study. Lancet Public Health 2020;5:e444-51. 